\title{
Huge Tracheal Diverticulum in a Patient with Mounier-Kuhn Syndrome
}

\author{
Michele Mondoni ${ }^{1}$, Paolo Carlucci ${ }^{1}$, Elena Maria Parazzini ${ }^{1}$, Paolo Busatto ${ }^{2}$, Stefano Centanni ${ }^{1}$ \\ ${ }^{1}$ Respiratory Unit, San Paolo Hospital, Dept. of Scienze della Salute, Università degli Studi di Milano, Milan, Italy \\ UO Pneumologia, San Luca Hospital, USL Nordovest Toscana, Lucca, Italy
}

How to cite this article: Mondoni M, Carlucci P, Parazzini EM, Busatto P, Centanni S. Huge tracheal diverticulum in a patient with Mounier-Kuhn syndrome EJCRIM 2016;3:doi:10.12890/2016_000419

Conflicts of Interests: The Authors declare that there are no competing interests.

This article is licensed under a Commons Attribution Non-Commercial 4.0 License

\section{ABSTRACT}

Tracheal diverticulum is a rare benign entity. Tracheobronchomegaly (TBM), also known as Mounier-Kuhn syndrome, is a rare disorder characterized by marked dilation of the trachea and main bronchi, associated with thinning or atrophy of the elastic tissue. Because of the weakened trachea and increased intraluminal pressure related to chronic cough, some patients may develop mucosal herniation leading to tracheal diverticulosis. We report the case of a patient with TBM with a huge tracheal diverticulum, diagnosed by bronchoscopy and computed tomography with three-dimensional reconstruction. to our knowledge this is the largest tracheal diameter described in a patient affected by this syndrome.

\section{LEARNING POINTS}

- Tracheal diverticulum is a rare condition that should be considered in the presence of bronchopulmonary disorders characterized by chronic cough and repeated bronchial infection, such as Mounier-Kuhn syndrome.

- The differential diagnosis of tracheal diverticulum includes laryngocele, pharyngocele, Zenker's diverticulum, apical lung hernia and lung bullae.

- Bronchoscopy and CT scans with three-dimensional reconstruction are useful tools for diagnosing this rare condition.

\section{KEYWORDS}

Tracheal diverticulum; tracheocele; Mounier-Kuhn syndrome; bronchoscopy.

\section{CASE REPORT}

A 45-year-old black man was referred to our pulmonary department for dyspnoea and productive cough with purulent sputum. He had experienced several respiratory infections over the past 10 years. He was a current smoker with a 25 pack-year smoking history; no potentially noxious occupational exposure was detected.

The physical examination revealed decreased breath sounds with velcro rales at the lower fields of both lungs. Routine haematological blood tests were unremarkable; blood gas analysis revealed a modest hypoxaemia $\left(\mathrm{pH} 7.43, \mathrm{PO}_{2} 76 \mathrm{mmHg}, \mathrm{PCO}_{2} 38 \mathrm{mmHg}, \mathrm{HCO}_{3} 24\right.$ $\mathrm{mmol} / \mathrm{l}$, BE $2.1 \mathrm{mmol} / \mathrm{l})$. Pulmonary function tests showed a moderate restrictive pattern. The chest $\mathrm{x}$-ray revealed marked dilation of the trachea and suggested lung fibrosis in the lower lung fields. The patient underwent chest computed tomography (CT) that showed tracheobronchomegaly, bilateral upper lobe emphysema, and cystic bronchiectasis involving both lung bases (Fig. 1A-D). There was also evidence of a wide diverticulum in the posterolateral wall of the upper trachea, extending from D1 to D2 vertebra (Fig. $2 A-C)$. At the level of the diverticulum, the maximum transverse diameter of the trachea was $60.1 \mathrm{~mm}$ (Fig. 1A). The sagittal diameters of the right and left main 
bronchi were $24.2 \mathrm{~mm}$ and $23.1 \mathrm{~mm}$, respectively.

The clinical presentation and the radiological features led us to the diagnosis of tracheobronchomegaly (TBM), also known as MounierKuhn syndrome ${ }^{[1,2]}$. Subsequent bronchoscopy confirmed the presence of wide central airways associated with flaccid walls with a tendency toward collapse upon expiration. The examination clearly highlighted the presence of a large outpouching in the upper trachea (Fig. $3 A-C$ ). Within the diverticulum, the mucosa seemed normal without retained secretions or blood. Culture tests of the aspirated mucopurulent bronchial secretions yielded no growth. The patient was successfully treated with antibiotic therapy (ceftriaxone) and was then transferred to a pulmonary rehabilitation programme to improve secretion cleaning and drainage. He was also advised to give up smoking.

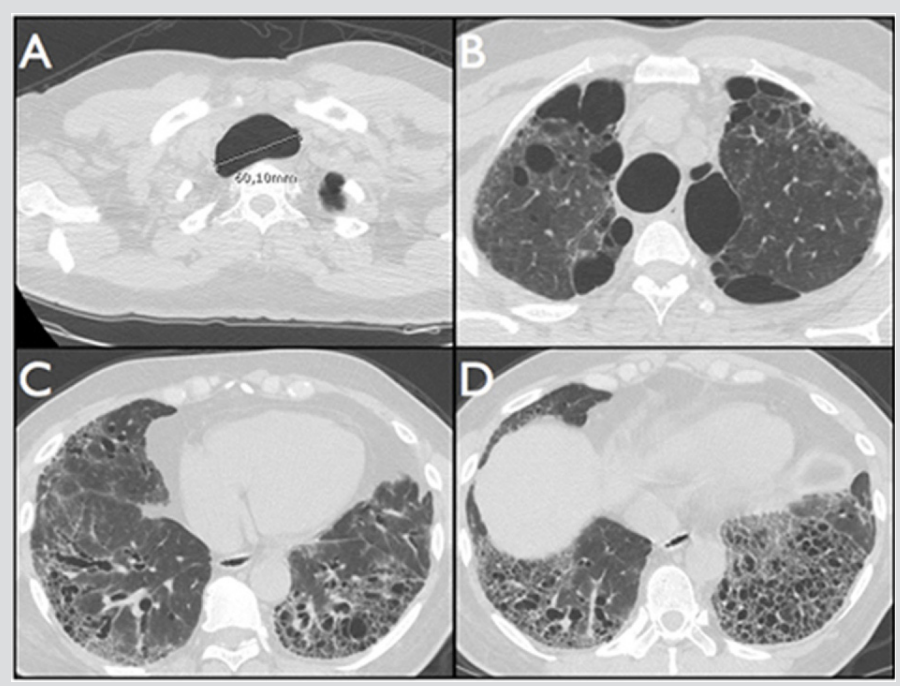

Figure 1. CT scans showing tracheomegaly (A), bilateral upper lobe emphysema (B) and cystic bronchiectasis involving both lung bases (C, D).

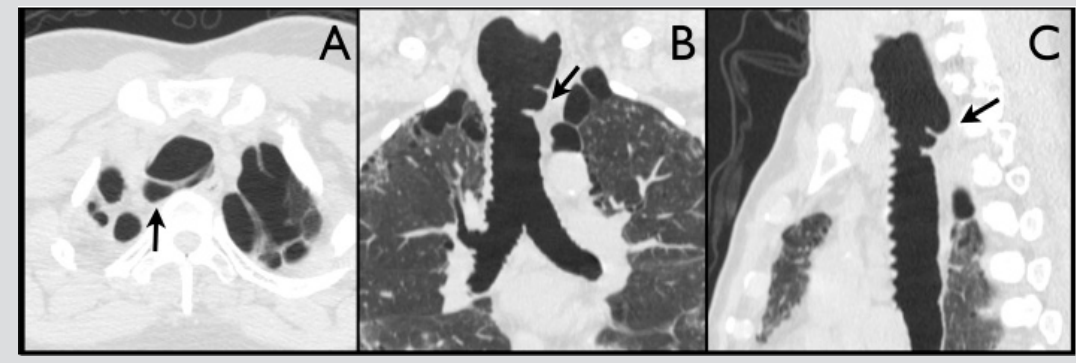

Figure 2. Axial (A), coronal (B) and sagittal (C) CT scans of the tracheal diverticulum (arrows).

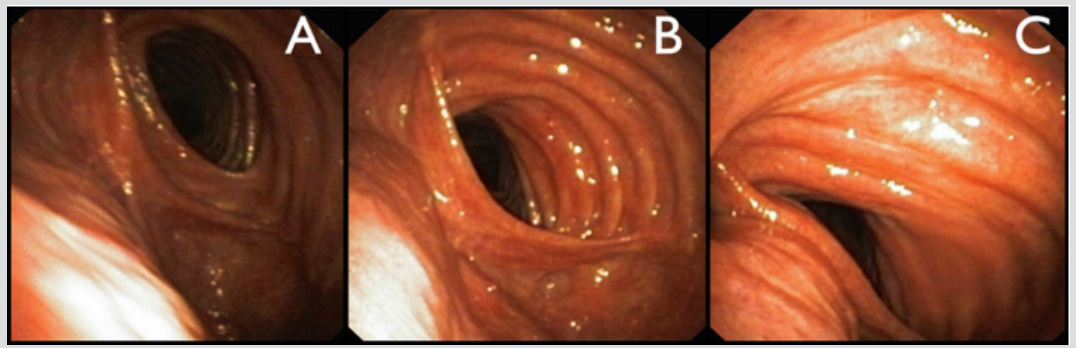

Figure 3. Bronchoscopic views of the tracheal outpouching, during inspiration $(A, B)$ and expiration $(C)$.

\section{DISCUSSION}

To our knowledge, this is the largest tracheal diameter described in a patient with TBM.

Tracheal diverticulosis is a rare entity that can be congenital or acquired. Usually only single congenital diverticula are found and their pathogenesis remains unclear. Acquired diverticula may arise at any level of the organ but are more frequent along the posterolateral wall between the junction of the extrathoracic and intrathoracic tracheae, probably as a result of an anatomical wall defect at this level. 
As in this case, diverticula mainly develop in the right paratracheal region where they receive no structural support from the oesophagus. The mechanism of development is related to increased intraluminal pressure and mucosal herniation through a weakened portion of the bronchial wall, as occurs in diseases characterized by chronic cough ${ }^{[3]}$.

Acquired tracheal diverticula are typically larger than congenital diverticula and have a wider opening. Although tracheal diverticula are usually classified as either congenital or acquired, other Authors have discriminated between tracheoceles and diverticula, with the former being one single formation or else larger than $2 \mathrm{~cm}$ and diverticula being multiple formations or smaller than $2 \mathrm{~cm}^{[3,4]}$.

Surprisingly, we described one single huge tracheocele in a patient with Mounier-Kuhn syndrome, a disease that is usually characterized by multiple tracheal diverticula ${ }^{[1,3,4]}$.

TBM is a rare disorder characterized by marked dilation of the trachea and main bronchi associated with thinning or atrophy of the elastic tissue $^{[1]}$. Six clinical sub-types of disease have been identified on the basis of the underlying aetiology. The presence of several reported pulmonary infections over the past decade led us to diagnose in this patient type 2A TBM, a clinical sub-type of the disease with less than 20 cases described in the literature ${ }^{[2]}$.

Although some patients may be clinically asymptomatic, cough and purulent sputum production are often present, as in the patient described here. Enlarged but weakened airways and an inefficient cough mechanism impede effective mucociliary clearance and lead to mucus retention, resulting in recurrent lower respiratory infections and bronchiectasis ${ }^{[3,4]}$. This situation may be exacerbated in a patient with a tracheal diverticulum that may act as a reservoir of secretions ${ }^{[3]}$. Notably, in tracheal diverticula cough may be either an aetiological factor or a symptom secondary to the lesion ${ }^{[4]}$.

The finding of a collection of paratracheal air first requires determination of the origin and size of the lesion ${ }^{[3]}$. The differential diagnosis includes laryngocele, pharyngocele, Zenker's diverticulum, apical lung hernia and bullae. Barium studies, CT and bronchoscopy may help identify the origin. Bronchoscopy is a useful tool in confirming the diagnosis, although there are cases in which the communication between the air cyst and the trachea is too narrow to be visible ${ }^{[3-5]}$. In the case described here, bronchoscopy allowed us to easily detect the opening of the evagination and to note expansion during inspiration and a partial collapse during expiration of the posterior wall of the trachea and of the diverticulum (Fig. $3 \mathrm{~A}-\mathrm{C}$ ). As suggested by other Authors, because of the predominantly intrathoracic localization, the negative intrapulmonary pressure during inspiration is transmitted to the weakened trachea, thus enlarging its size ${ }^{[4]}$.

When the diagnosis remains uncertain, three-dimensional reconstruction CT and virtual bronchoscopy may help identify the connection between the diverticulum and the tracheal lumen and define the extent of the lesion, especially in the presence of numerous outpouchings (Fig. 4A-C) ${ }^{[5]}$.

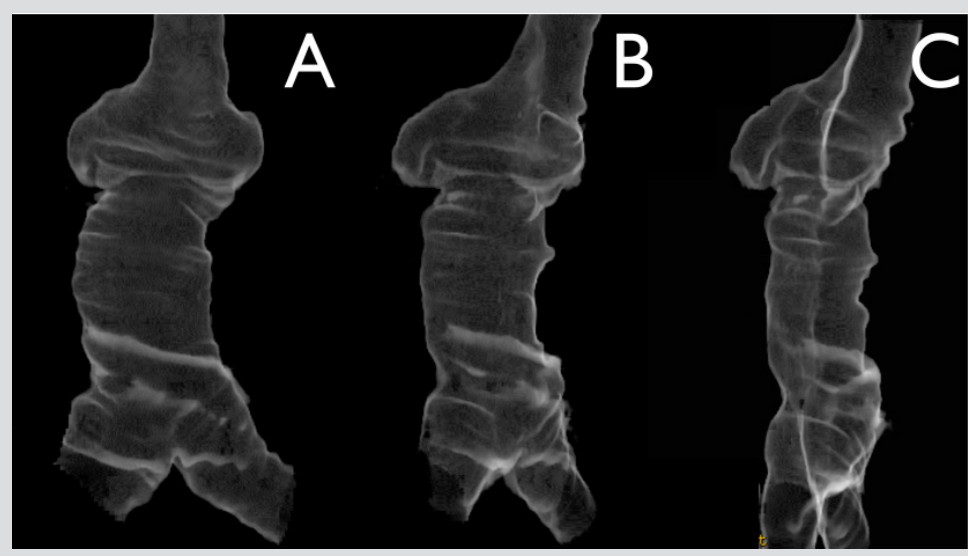

Figure 4. Three-dimensional $C T$ reconstruction of the trachea with the diverticulum. Frontal (A) and right anterolateral views (B, C) at different rotation angles.

Treatment options may be surgical or medical, depending on the patient's physical state, age and symptoms. Surgical options should be reserved for young patients. Nevertheless, the majority of cases are asymptomatic and do not require any specific intervention. In symptomatic cases, antibiotics, mucolytic agents and physiotherapy, as we prescribed for our patient, represent the treatment of choice [3]. In conclusion, tracheal diverticulum is a rare condition that should always be considered in the presence of bronchopulmonary disorders characterized by chronic cough and repeated bronchial infection, such as TBM. Bronchoscopy and CT scans with three-dimensional reconstruction are useful tools to diagnose this rare condition. 


\section{REFERENCES}

1. Schwartz M, Rossoff L. Tracheobronchomegaly. Chest 1994:106:1589-1590.

2. Payandeh J, McGillivray B, McCauley G, Wilcox P, Swiston JR, Lehman A. A clinical classification scheme for tracheobronchomegaly (Mounier-Kuhn syndrome). Lung 2015;193:815-822.

3. Soto-Hurtado EJ, Peñuela-Ruiz L, Rivera-Sanchez I, Torres-Jimenez J. Tracheal diverticulum: a review of the literature. Lung 2006;184:303-307.

4. Rojas J, Bostanci K, Katsenos S, Becker HD. Tracheal diverticulum. J Bronchol Intervent Pulmonol 2011;18:91-93.

5 Polverosi R, Carloni A, Poletti V. Tracheal and main bronchial diverticula: the role of CT. Radiol Med 2008;113:181-189. 